\title{
Modeling and Simulation of Synchronous Threshold in Vent Collective Behavior
}

\author{
Yaofeng Zhang ${ }^{1,2}$ and Renbin Xiao ${ }^{1}$ \\ ${ }^{1}$ School of Automation, Huazhong University of Science and Technology, Wuhan 430074, China \\ ${ }^{2}$ Statistic College, Hubei University of Economics, Wuhan 430205, China
}

Correspondence should be addressed to Renbin Xiao; rbxiao@hust.edu.cn

Received 10 January 2014; Revised 15 April 2014; Accepted 16 April 2014; Published 15 May 2014

Academic Editor: Manuel De la Sen

Copyright (c) 2014 Y. Zhang and R. Xiao. This is an open access article distributed under the Creative Commons Attribution License, which permits unrestricted use, distribution, and reproduction in any medium, provided the original work is properly cited.

With the strengthening of the social contradiction, the outbreak of vent collective behavior tends to be frequent. The essence of vent collective behavior is emergence of synchronization. In order to explore the threshold of consensus synchronization in vent collective behavior, a mathematic model and a corresponding simulation model based on multi-agent are proposed. The results of analysis by mean field theory and simulation experiments show the following. (1) There is a threshold $K_{c}$ for consensus synchronization in global-coupling and homogeneous group, and when the system parameter $K$ is greater than $K_{c}$, consensus synchronization emerge. Otherwise the system cannot achieve synchronization. The conclusion is verified by further study of multiagent simulation. (2) Compared with the global-coupling situation, the process of synchronization is delayed in local-coupling and homogeneous group. (3) For local-coupling and heterogeneous group, consensus dissemination can achieve synchronization only when the effects of the parameters meet the threshold requirements of consensus synchronization.

\section{Introduction}

Synchronous behaviors formed by self-organization are ubiquitous in nature $[1,2]$. A large number of physical and biological systems can be thought of as a synchronous system. The most striking visual example is given by the synchronous flashing of fireflies observed in summer season in some regions of South Asia [3], and more examples include chorusing crickets [4] and coupled laser arrays [5]. One interesting common phenomenon in all these systems is their ability to synchronize: a large number of the individuals lock onto a common frequency.

In fact, the synchronous behaviors are widespread not only in the biological world, but also in society, such as the synchronization applause in music hall [6]. Sometimes, some burst factors cause people to vent their emotions. Under some invisible "cohesion," they participate in a temporary group by self-organization, and, then, synchronous behavior may be formed. We call the synchronous behavior as vent collective behavior [7]. As a social phenomenon, how does the infection of emotions and consensus work in the group composed of independent individuals? And, through individuals' consensus transmission and behavior imitation, how does the synchronous behavior emerge? Is there a synchronous threshold in vent collective behavior?

So far, there has been very little work on the above questions. This is due to the complexity of vent collective behavior. It is difficult to establish a model corresponding with the actual situation by quantitative analysis. In recent years, with the in-depth study of social complex systems, related topics begin to arouse numerous scholars' interests in different fields, such as sociology, psychology, computer science, and physics, but what they care about is different. For sociologists [8] and psychologists [9], they qualitatively analyze the problem from the political angle, computer scientists [10] are concerned about the simulation of collective escape behavior under the emergency, and physicists [11] are more willing to study the synchronization in complex network.

With the development of complex system science, simulation based on multiagents is more and more popular as a new research method [12-14]. Some scholars begin to use it to study the consensus transmission in collective behaviors. The early simulation researches made individuals in collective behavior homogeneous [15]. After that, researches on the 


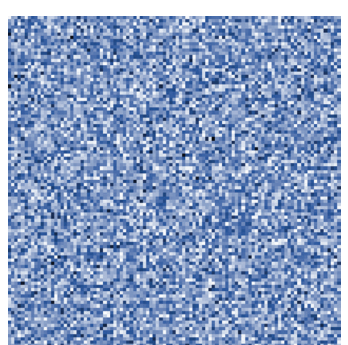

(a) $c_{0}=0.3 ; \sigma^{2}=0.17$

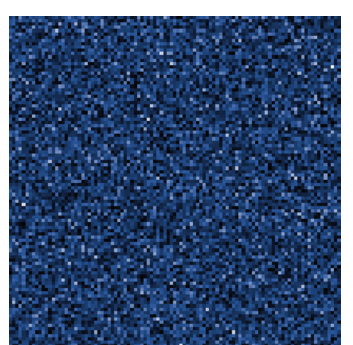

(b) $c_{0}=0.7 ; \sigma^{2}=0.17$

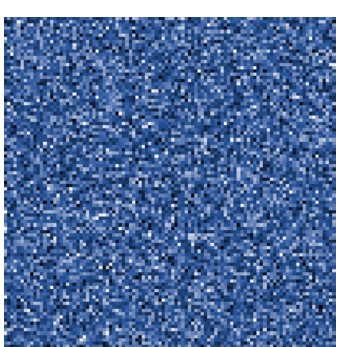

(c) $c_{0}=0.5 ; \sigma^{2}=0.17$

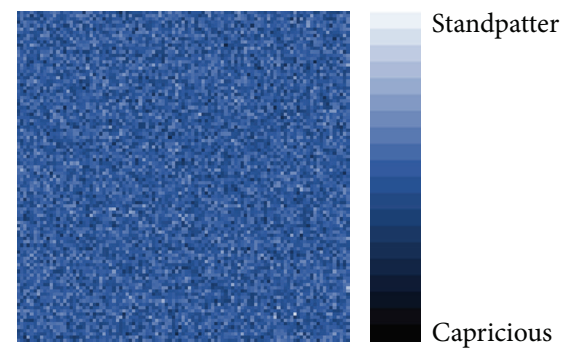

(d) $c_{0}=0.5 ; \sigma^{2}=0.08$

FIGURE 1: Influence of parameters in normal distribution on individual's attitude; $0 \leq c \leq 1$. Light color expresses individual is a standpatter (more inclined to keep its attitude). Dark color expresses individual is capricious (more inclined to change its attitude). The influence of mathematical expectation on individual's initial conformity is shown in (a) and (b). The influence of variance on individual's conformity is shown in (c) and (d).

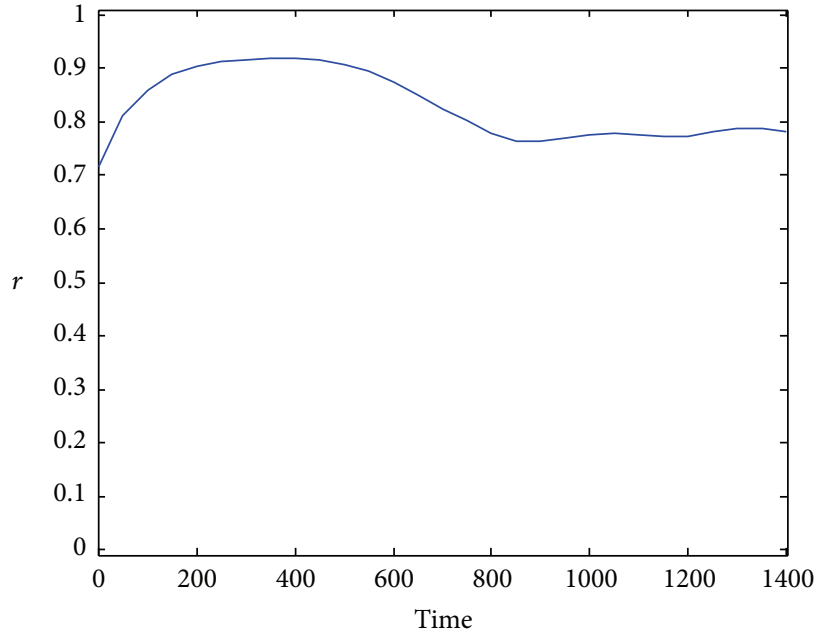

(a)

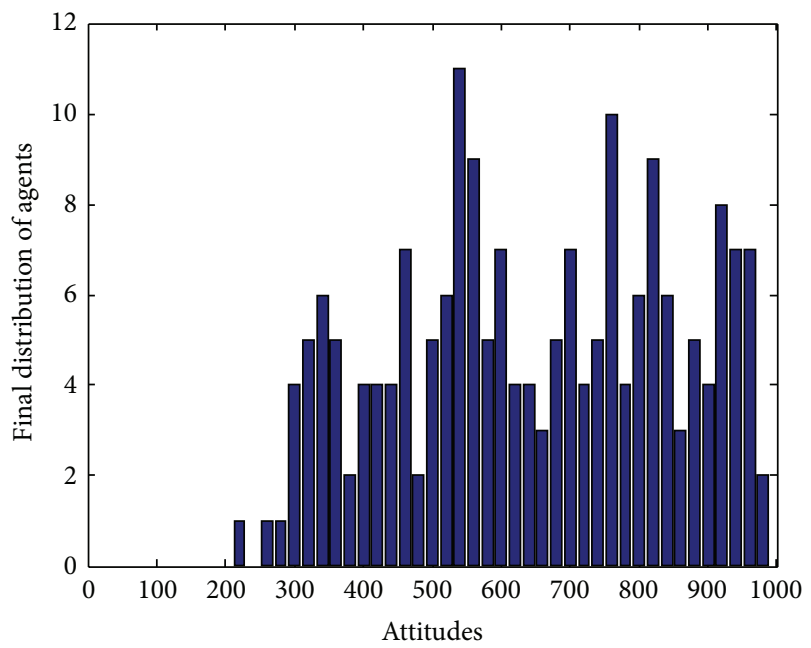

(c)

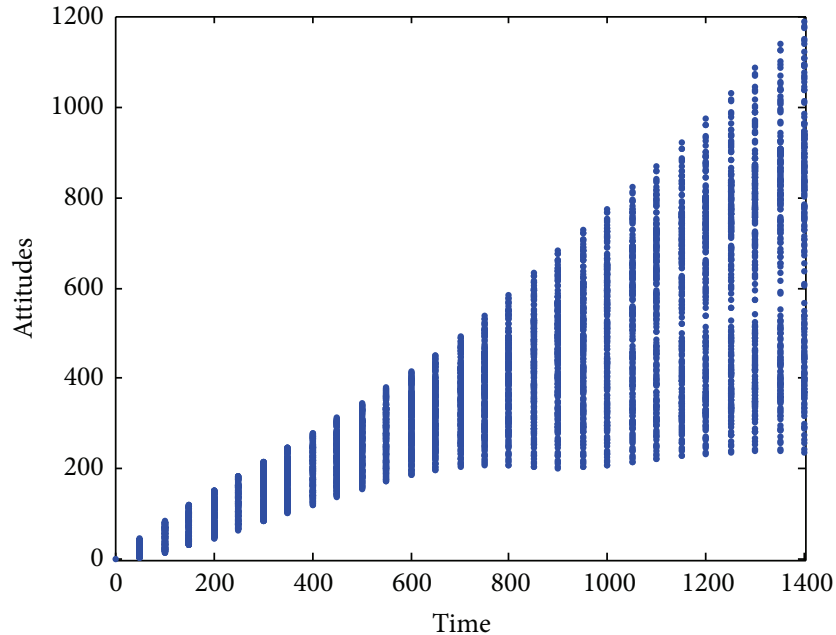

(b)

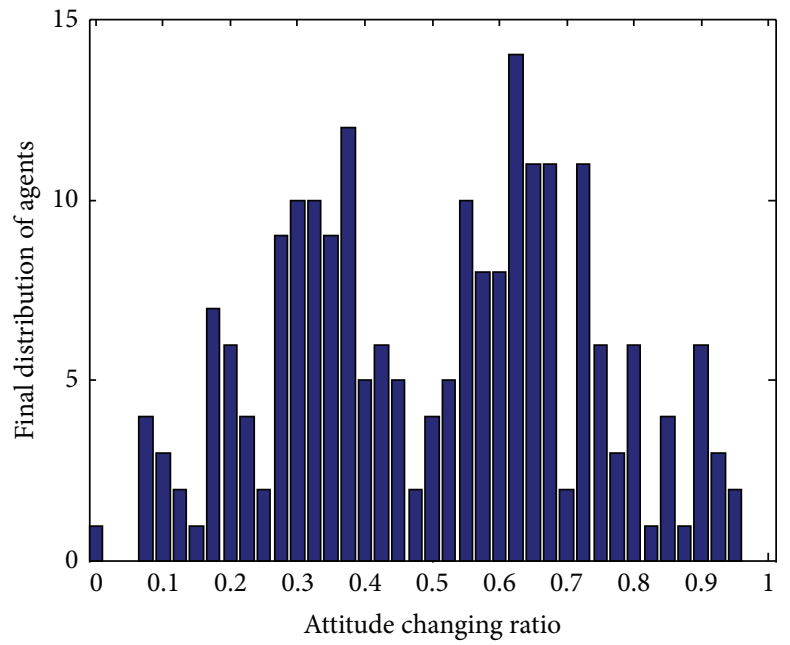

(d)

FIGURE 2: Results of simulation system with $K=0.5$. (a) is the subfigure which shows synchronization criterion $r$ changes with time; (b) is the subfigure which shows group attitudes $x(t)$ versus time; (c) is the histogram of the final distribution of agents versus group attitudes $x(t)$; (d) is the histogram of the final distribution of agents versus attitude changing ratio $\dot{x}_{i}(t)$. 


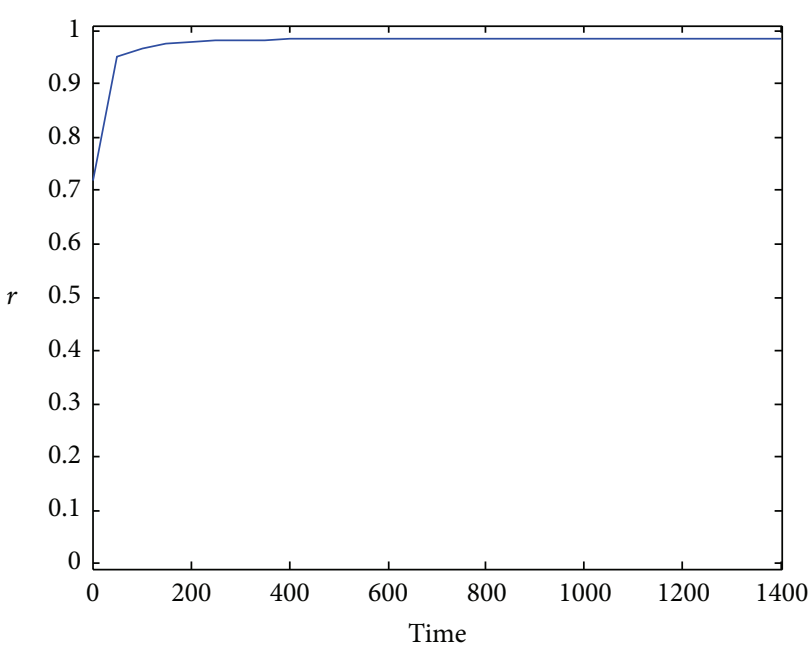

(a)

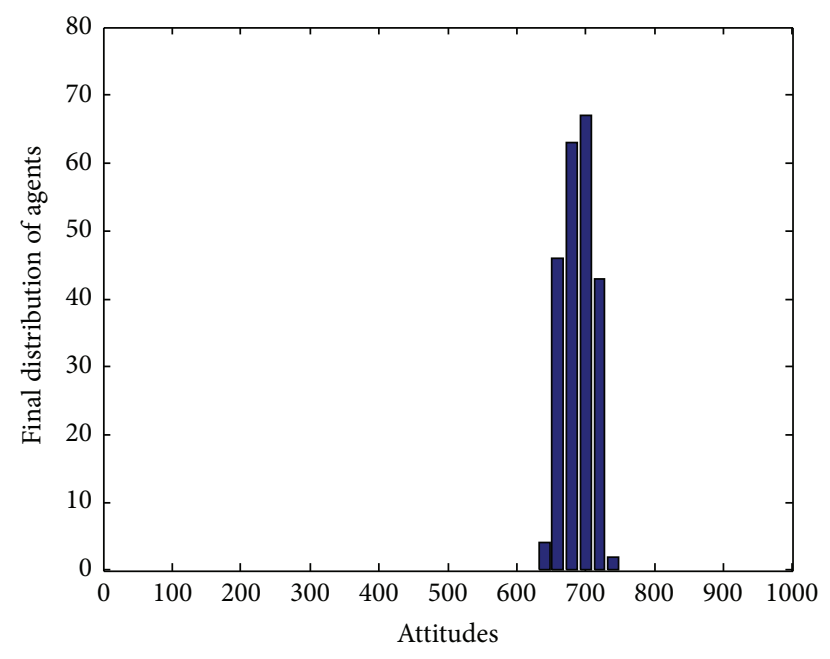

(c)

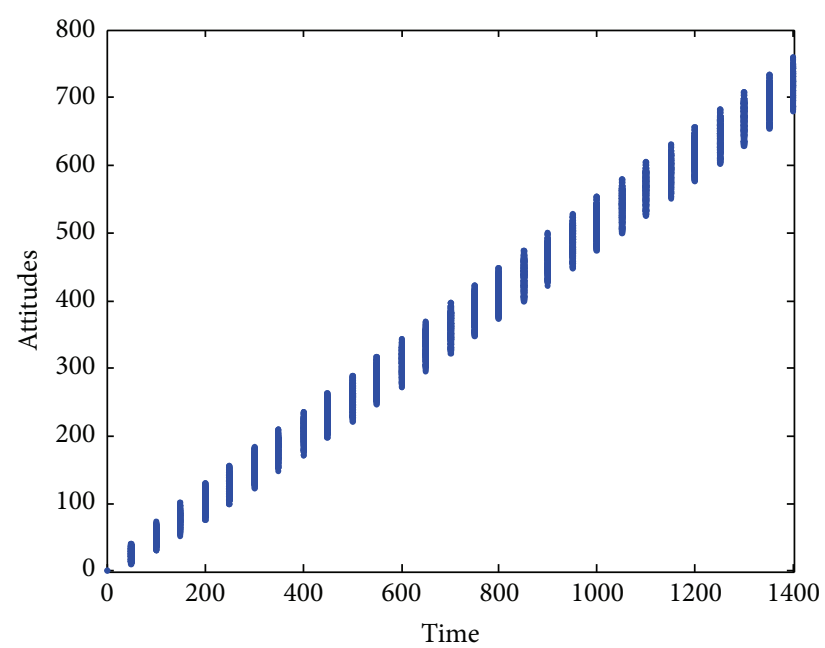

(b)

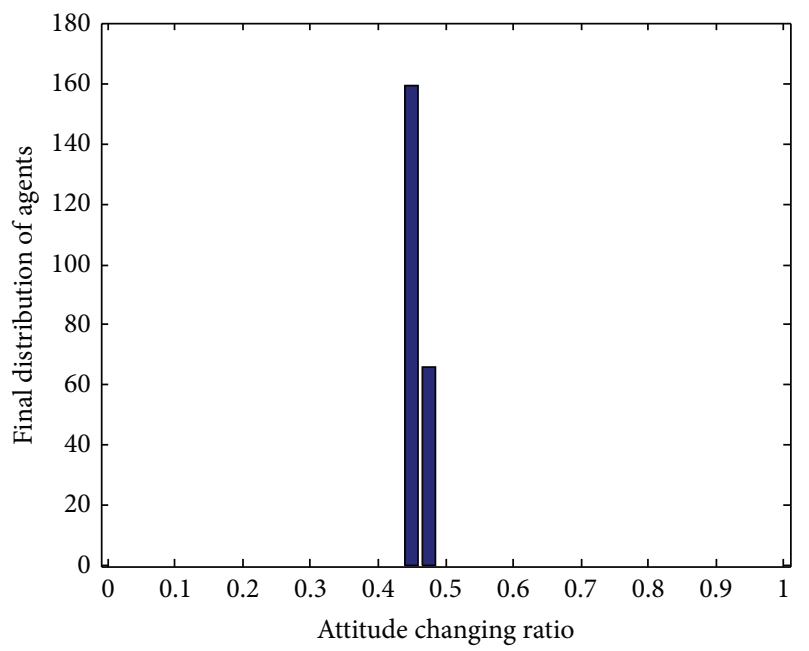

(d)

FIGURE 3: Results of simulation system with $K=1$. (a) is the subfigure which shows synchronization criterion $r$ changes with time; (b) is the subfigure which shows group attitudes $x(t)$ versus time; (c) is the histogram of the final distribution of agents versus group attitudes $x(t)$; (d) is the histogram of the final distribution of agents versus attitude changing ratio $\dot{x}_{i}(t)$.

effects of various characteristics on collective behavior were developed [16]. Recently, some scholars used continuous view dynamic model [17] to study the formation dynamics of consensus in collective behavior. They took the real number from 0 to 1 to represent the individual's opinions on social events. Through the simulation experiments, they observed rich evolution dynamic phenomenon.

In fact, in the collective behavior, individuals learn from each other and then make the strategy choice in the next time step. In this process, each individual just can be seen as an agent who has ability to learn from others and "think" of its future strategy. And then, the group can be regarded as a complex system with multiagents.

Motivated by the above work $[6,15-17]$ and the discussions earlier, in this paper, we are concerned with the problem of synchronous threshold in vent collective behavior. In Section 2, we set up a model of consensus dissemination of vent collective behavior. The model is analyzed by mean field method [18] in Section 3, and the common conclusions of the synchronous threshold in vent collective behavior are given. For more complex situation of local-coupling and homogeneous group, multiagent simulation model based on sociology and psychology is proposed in Section 4. We will put all individuals in a two-dimensional grid space and define that each individual has heterogeneous conformity feature, attitude, and influence ability. The attitude of the individual to the social events is influenced by the whole group consensus or near neighbors' opinion. Through a series of simulation experiments in Section 5, we will prove that there is a threshold which induces the synchronous consensus in collective behavior, and the synchronous consensus is delayed in the local-coupling environment than global-coupling with the same parameters. For local-coupling and heterogeneous group, consensus dissemination can achieve synchronization 


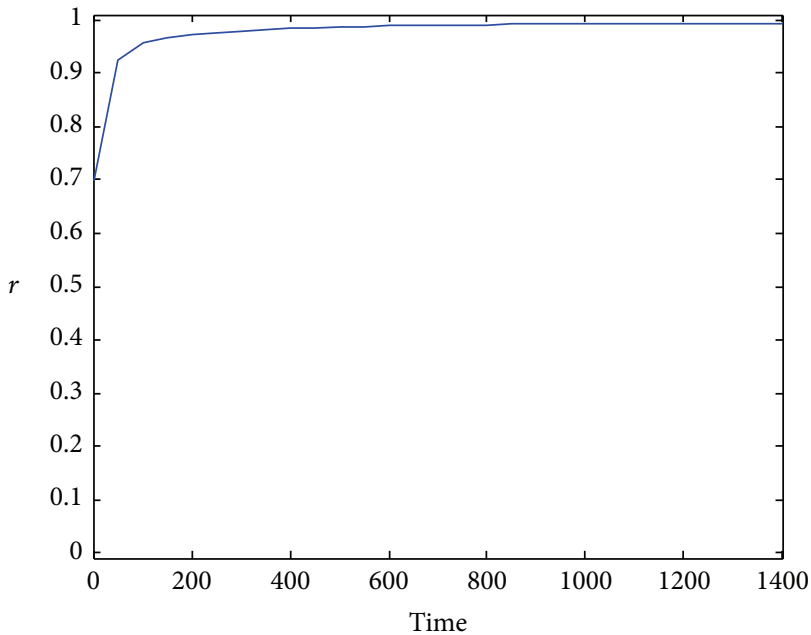

(a)

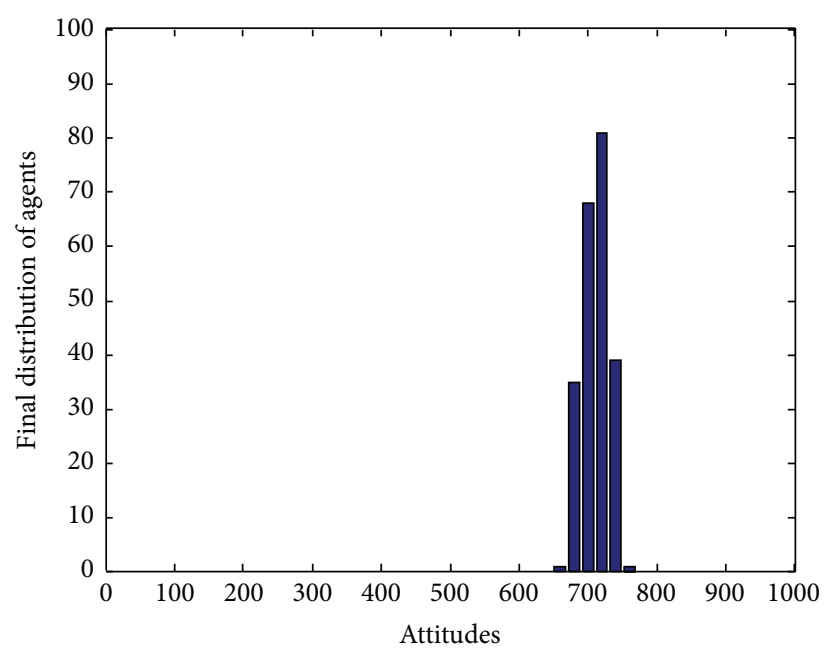

(c)

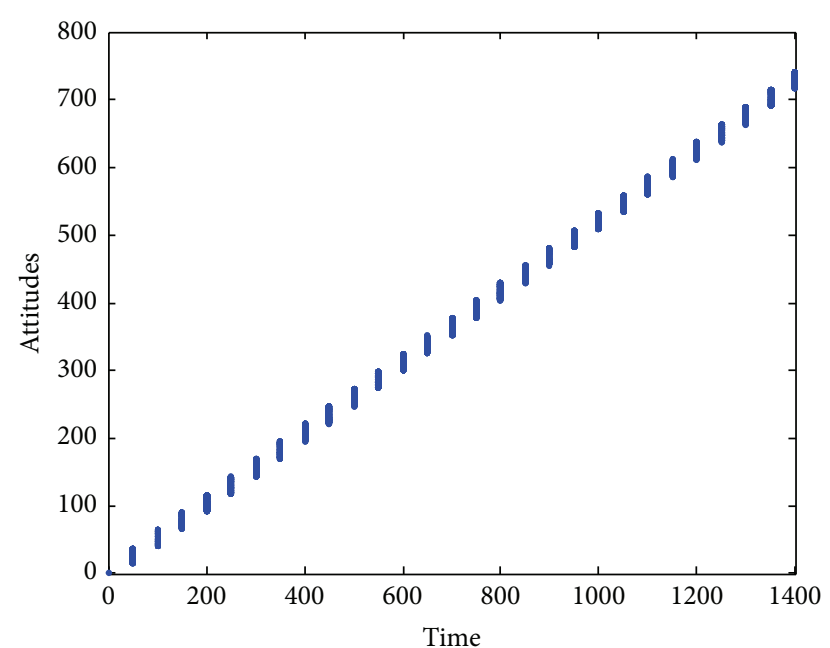

(b)

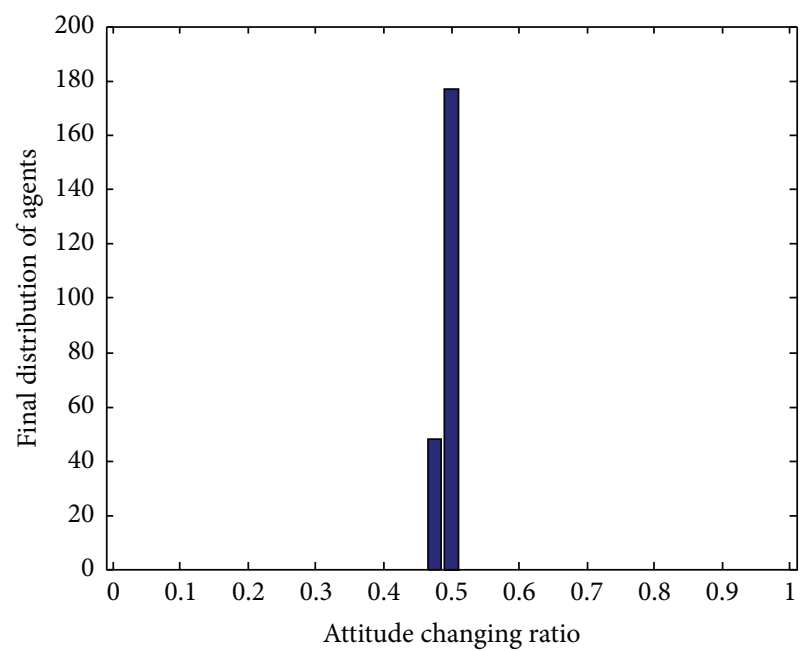

(d)

FIGURE 4: Results of simulation system with $K=2$. (a) is the subfigure which shows synchronization criterion $r$ changes with time; (b) is the subfigure which shows group attitudes $x(t)$ versus time; (c) is the histogram of the final distribution of agents versus group attitudes $x(t)$; (d) is the histogram of the final distribution of agents versus attitude changing ratio $\dot{x}_{i}(t)$.

only when the effects of the parameters meet the threshold requirements of consensus synchronization.

\section{Consensus Dissemination Model}

In vent collective behavior, most participants are not direct gainers who participate in collective behavior not due to benefits but just venting their emotions. Different from direct gainers who have direct interest in the event, they have no direct relationship with event, and they also do not know each other, so they are relatively independent. Nevertheless, each individual has been infected by group consensus to adjust its attitude of whether to participate in the behavior. Because vent collective behavior bursts out generally and has a short duration, we assume that the number of participants is $N$, and each individual $i$ has relatively stable $\Omega_{i}$ neighbors for the great crowd density. Normally, when a person is faced with an event, he has the attitude which will decide his behavior strategies. So we assume that individual $i$ 's attitude is $x_{i}(t)$ at time $t$. Clearly the evolution of individual's attitude is influenced by its attitude in the previous stage and coupling effect between individuals, where the coupling effect between individuals contains two aspects: external impact on the individual and individual's acceptance of external impact.

Firstly, we consider the external impact on the individual. Because each individual has a different number of neighbors in vent collective behavior, we set that the individual $i$ has $n_{i}$ neighbors. Since each individual's ability of influencing others to change attitudes is different, we set that $\alpha_{j}$ represents individual $j$ 's influence on others, which is different caused by the difference of abilities, such as expressive ability. Take that $\alpha$ obey the normal distribution function $h(\alpha)$. Considering that the opinion of individuals is affected not only by their 


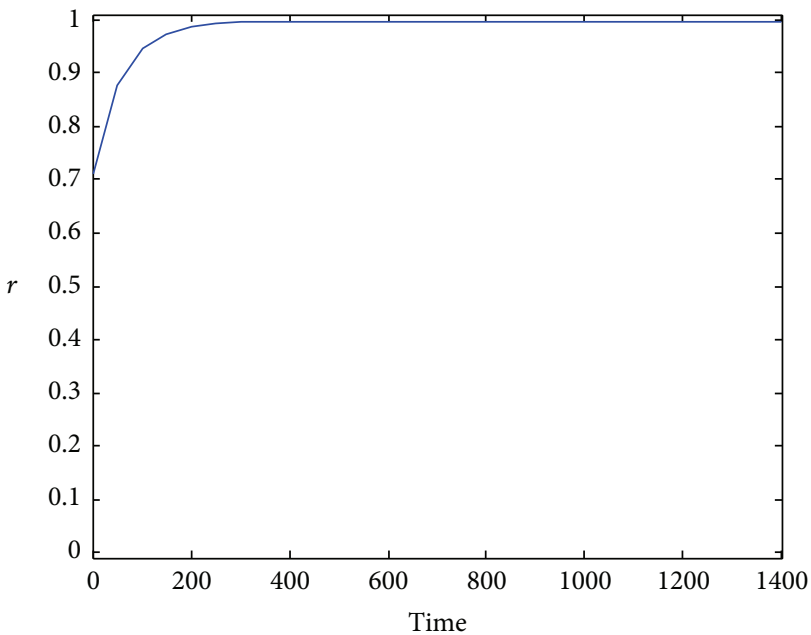

(a)

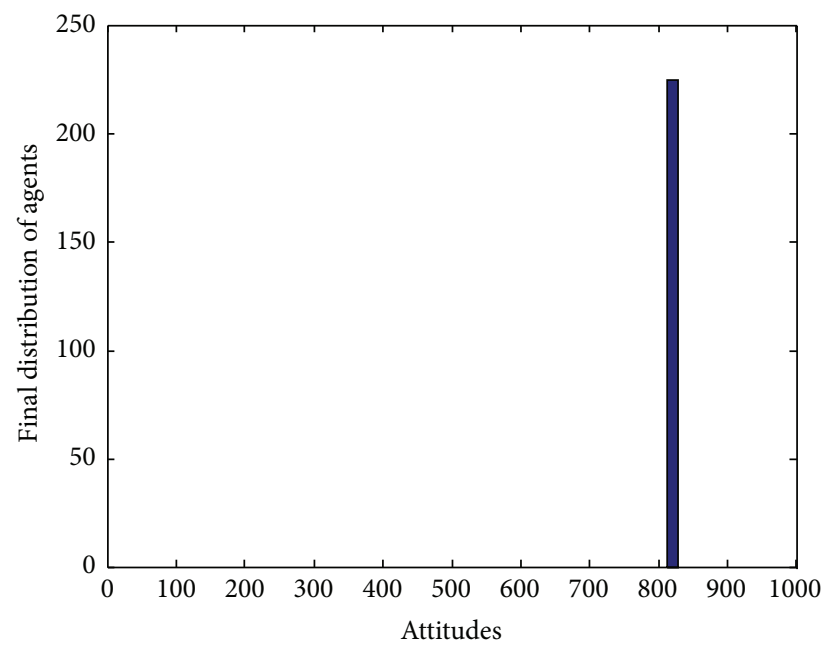

(c)

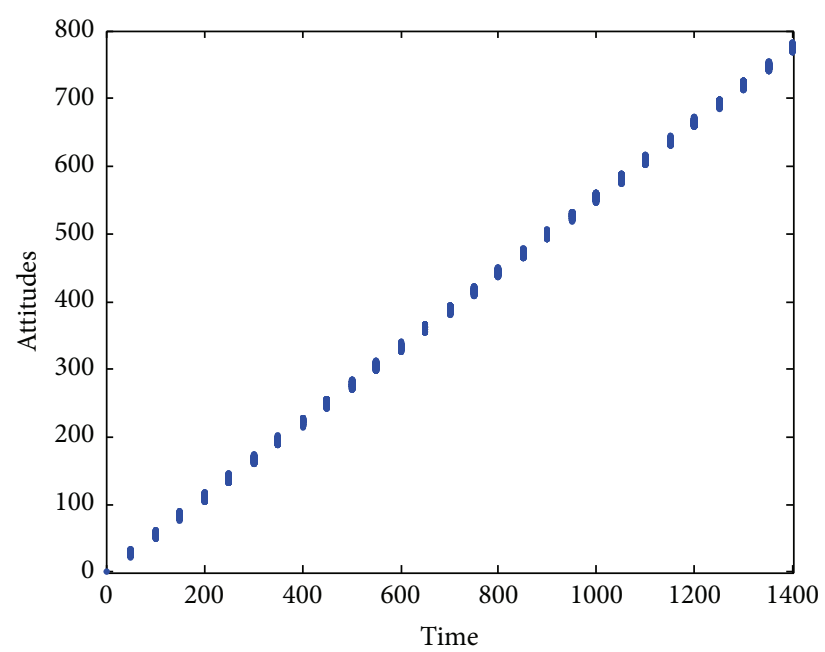

(b)

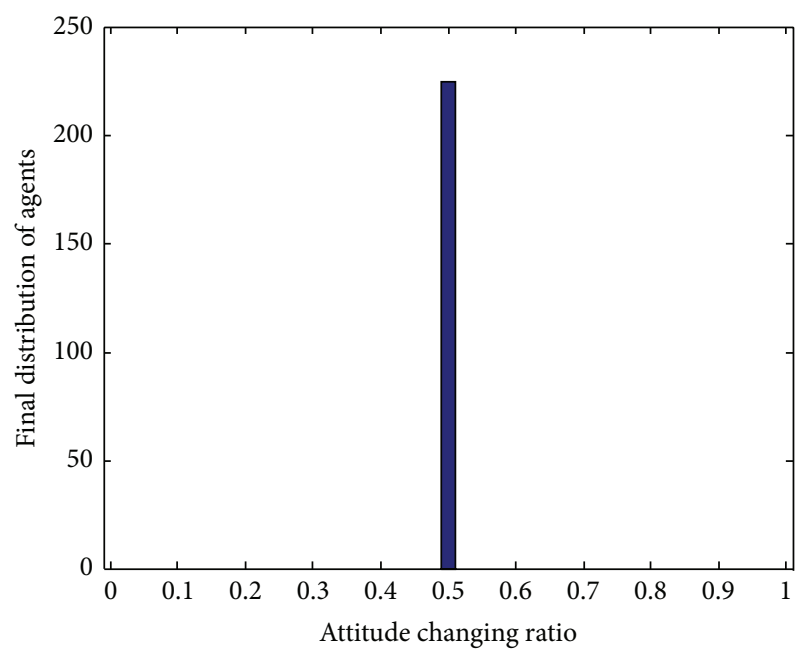

(d)

FIGURE 5: Results of simulation system with $K=5$. (a) is the subfigure which shows synchronization criterion $r$ changes with time; (b) is the subfigure which shows group attitudes $x(t)$ versus time; (c) is the histogram of the final distribution of agents versus group attitudes $x(t)$; (d) is the histogram of the final distribution of agents versus attitude changing ratio $\dot{x}_{i}(t)$.

neighbors but also by the opinion of themselves, we express the individual $j$ 's influence on neighbor $i$ as

$$
\alpha_{j} H\left[x_{j}(t)-x_{i}(t)\right],
$$

where $H$ is the attitudes influence function. $H\left[x_{i}(t)-x_{i}(t)\right]$ reflects the differences between individuals' opinion and their neighbors' opinion; the greater the difference the greater the impact on the individual and the converse is also true.

When $x_{j}>x_{i}$, individual $j$ is more inclined to participate in vent collective behavior than individual $i$, and the converse is also true. Individual $j$ 's influence on individual $i$ will be greater when $\alpha_{j}$ is greater. We use the mean value of all neighbors' influence to represent the combined influence on individual $i$, namely,

$$
\frac{1}{n_{i}} \sum_{j \in \Omega_{i}} \alpha_{j} H\left[x_{j}(t)-x_{i}(t)\right] .
$$

Secondly, we consider individual's acceptance for external impact. Although the individual will be influenced by group consensus to adjust its own attitude, the change degree of each individual is different. To characterize this feature, we set that $w_{i}$ shows individual's ability of accepting influence. $w_{i}$ is a random value of a normal distribution function $f(w)$. In addition, individual's conformity, namely, frequency of changing its attitude, is different for the external impact. We set that $c$ denotes individual's conformity coefficient. The $c$ obeys the normal distribution function $g(c)$.

Therefore, individual's attitude in vent collective behavior can update as the following formula:

$$
\begin{array}{r}
\dot{x}_{i}(t)=c_{i}+w_{i} \cdot \frac{1}{n_{i}} \sum_{j \in \Omega_{i}} \alpha_{j} H\left[x_{j}(t)-x_{i}(t)\right] \\
(i=1,2, \ldots, N),
\end{array}
$$




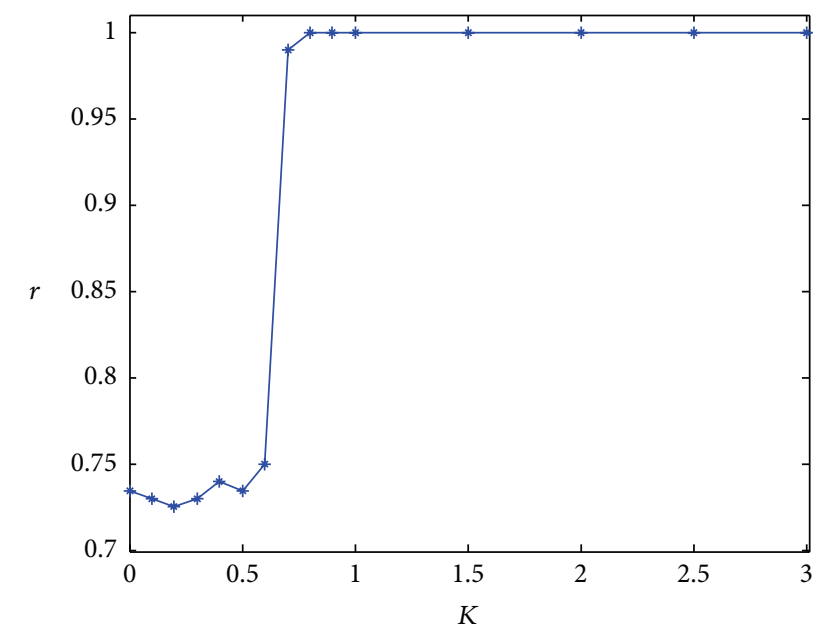

FIGURE 6: When group is global-coupling, the threshold $K_{c}$ is about 0.6 for consensus synchronization in vent collective behavior.

where $x_{i}, w_{i}$, and $\alpha_{j}$ are all real numbers. Formula (3) shows that individual's attitude is influenced by its attitude in the previous stage, its conformity coefficient, and the average influence of its neighbors. Individual's attitude update rule considers not only individual's receptivity, but also individual's infective ability, so it can represent the coupling influence among individuals in vent collective behavior.

\section{Synchronous Threshold in Vent Collective Behavior}

In fact, because there are heterogeneous neighbors (localcoupling) and heterogeneous parameters in model (3), we cannot get the analytic solution of this complex model; to find the threshold of synchronization in vent collective behavior, let us consider one special case firstly.

In global-coupling group $\left(n_{i}=N\right)$, we set $K=w_{i} \alpha_{j}$, where $K$ is a constant. Consider that, in vent collective behavior, individuals maybe change their attitudes frequently. For convenience, we assume that $H$ is a periodic function, and $H\left[x_{j}(t)-x_{i}(t)\right]=\sin \left[x_{j}(t)-x_{i}(t)\right]$. So formula (3) is essentially the Kuramoto model which shows that there is a coupling strength threshold which can lead to synchronization of a set of oscillators with fixed amplitude mutually coupled by a $2 \pi$ periodic interaction [19]. In recent years, various extensions and generalizations models based on Kuramoto model have been analyzed more deeply [20]. However, in the original form of this model, the individuals are global-coupling and parameter $K$ is homogeneous.

By constructing the order parameter as [21]

$$
r e^{i \bar{x}}=\frac{1}{N} \sum_{j=1}^{N} e^{i x_{j}}
$$

then formula (3) can be rewritten as

$$
\dot{x}_{i}(t)=c_{i}+K r \sin \left[\bar{x}-x_{i}(t)\right] \quad i=1,2, \ldots, N,
$$

where $\bar{x}$ is the average value of all individuals' attitudes.
Supposing the density function of $x$ is $\rho(x, c, t)$ in a limited population, the density function $\rho(x, c, t)$ accords with the conditions [22]

$$
\frac{\partial \rho}{\partial t}+\frac{\partial}{\partial x}[c+\rho K r \sin (\bar{x}-x)]=0
$$

Formula (5) shows that when individuals' attitudes incline to synchronization, $r$ is close to 1 , and when individuals' attitudes incline to dissimilarity, $r$ trails off.

Formula (6) shows that when the mutual influence is faintness among individuals, especially, $K \rightarrow 0$, the differential coefficient of $\rho(x, t)$ on time $t$ is 0 . Then from formula (3), it can be seen that $x_{i}(t)=x_{i}(0), \forall i=1,2, \ldots N$; namely, individual's attitudes $x_{i}(t)$ maintain its initial state, so synchronization cannot emerge in this situation. When the mutual influence is strong enough, especially, $K \rightarrow \infty$, equation has a balanced solution $\psi \approx x_{i}(t), \forall i$. Now, $r \approx 1$, and synchronization emerges.

Inspired by the above analysis, some people consider whether there is a threshold $K_{c}$ which will make attitudes achieve synchronization. According to the mean field theory [18], they find that when $r \rightarrow 0^{+}$, the threshold $K_{c}=2 / \pi g(0)$ will make individuals' attitudes achieve synchronization [20, 22].

This is to say, in global-coupling and homogeneous parameters case, when $K>K_{c}$, consensus of vent collective behavior will achieve partial or complete synchronization. When $K \leq K_{c}$, consensus cannot achieve synchronization. Then, can the same conclusion be got for more complex situation just as local-coupling case? And when we consider the heterogeneous conformity and influence ability of individuals, what laws will formula (3) show?

Because there is no analytic solution with local-coupling and heterogeneity parameters case, in the rest of the sections, we will set up multiagent simulation model in Section 4 and verify the conclusions of Section 3 in Section 5.1; then, we will study the more complex case of the model by multiagent simulation experiments in Sections 5.2 and 5.3.

\section{Multiagent Simulation Model}

We use agent to represent an independent person with the ability to make decisions and make two-dimensional grid the agent's environment which generally take $m$ line $n$ column, such as $20 \times 30$ or $50 \times 50$. Every agent occupies one node. The individual's attitude is related to both its conformity coefficient and consensus of vent collective behavior.

Definition 1. Agent $=\{A, C, \Omega, \Phi, X, t\}$, where

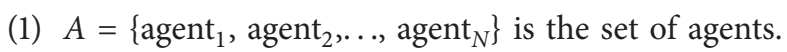
Every agent corresponds to an individual;

(2) $C=\left\{c_{1}, c_{2}, \ldots c_{N}\right\}$ is attitude changing ratio feature space. It is used to reflect the conformity of agents. Generally, $c_{i}$ is a constant. In the initial state, $c_{i}$ randomly comes forth according to normal distribution function $g(c)$ and does not alter with time;

(3) $\Omega=\left\{w_{1}, w_{2}, \ldots, w_{N}\right\}$ is accepting ability feature space. It is used to reflect the ability of accepting the 


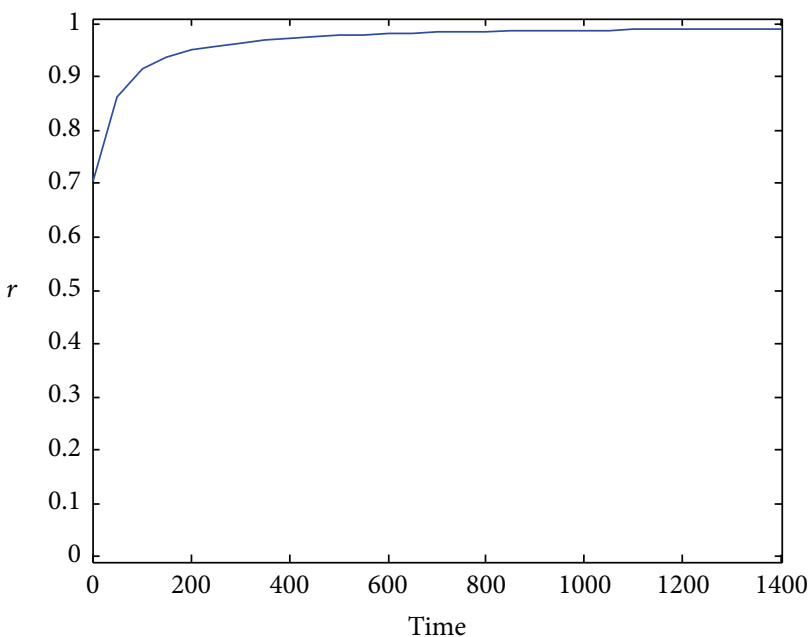

(a)

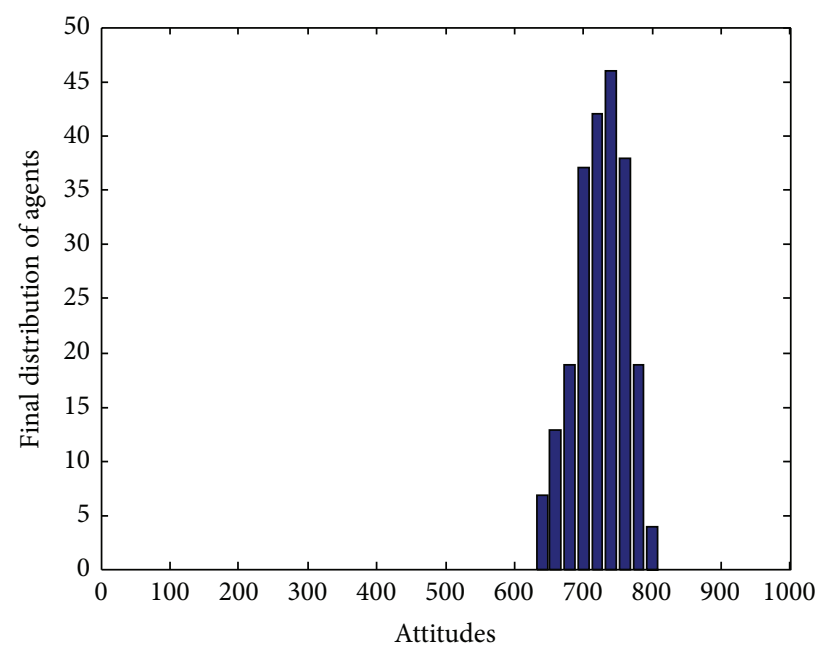

(c)

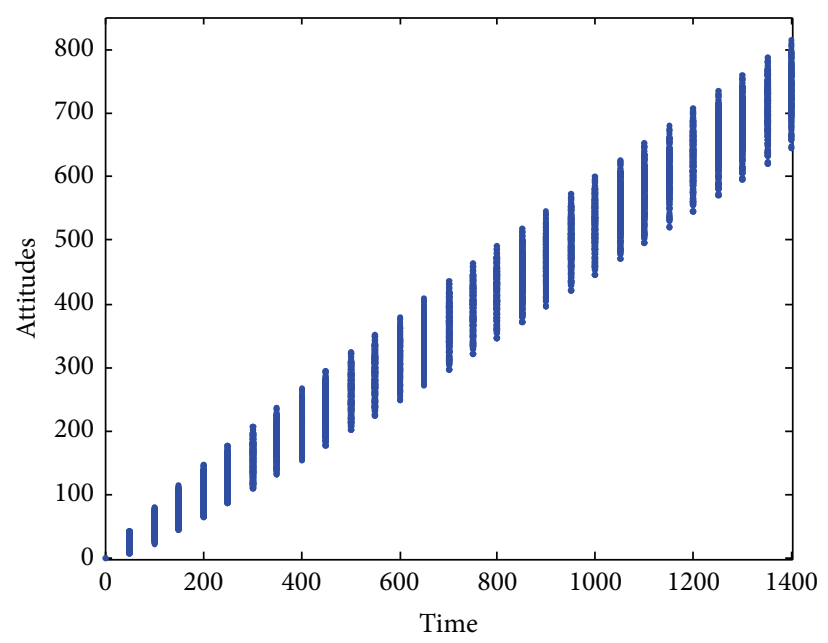

(b)

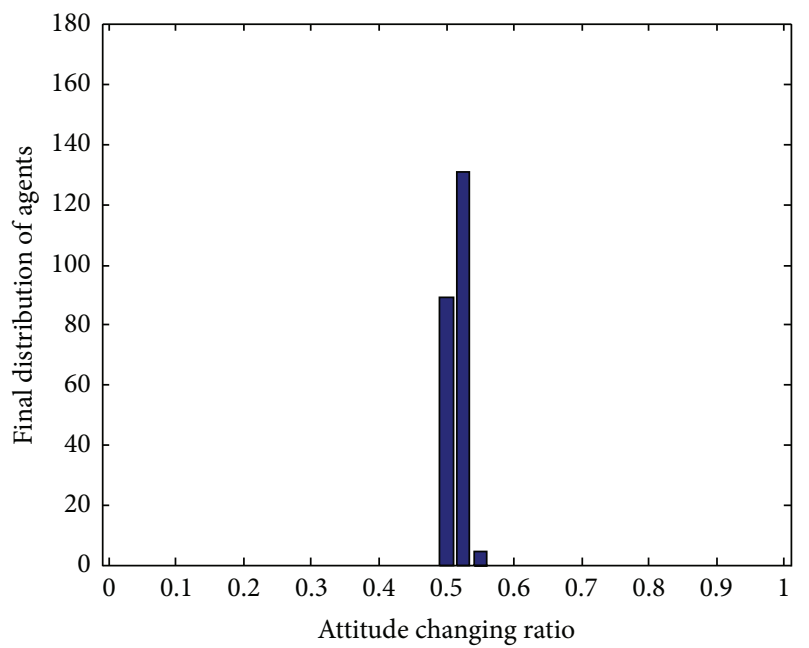

(d)

FIGURE 7: Results of simulation system with $K=1$. (a) is the subfigure which shows synchronization criterion $r$ changes with time; (b) is the subfigure which shows group attitudes $x(t)$ versus time; (c) is the histogram of the final distribution of agents versus group attitudes $x(t)$; (d) is the histogram of the final distribution of agents versus attitude changing ratio $\dot{x}_{i}(t)$.

influence of neighbors. Generally, $w_{i}$ is a constant. In the initial state, $w_{i}$ randomly comes forth according to normal distribution function $f(w)$ and does not alter with time;

(4) $\Phi=\left\{\alpha_{1}, \alpha_{2}, \ldots, \alpha_{N}\right\}$ is influence ability feature space. It is used to reflect the individual's ability of influencing others. Generally, $\alpha_{i}$ is a constant. In the initial state, $\alpha_{i}$ randomly comes forth according to normal distribution function $h(\alpha)$ and does not alter with time;

(5) $X=\left\{x_{1}(t), x_{2}(t), \ldots, x_{N}(t)\right\}$ is individual's attitude feature space, and $x(t)$ denotes individual's attitude for whether to participate in vent collective behavior, and it alters with time;

(6) $t=\{1,2,3 \ldots\}$ is system clock, which is the basis of the evolution of simulation system.
In the process of multiagent modeling, how to set up the effective individual interaction rule is a key problem. In social physics, what behavior strategy individual takes in vent collective behavior is a kind of group imitation action, and it is also a kind of gradual learning process. At present, there are a lot of evolutionary algorithms, such as the local evolutionary stable strategy and replicated dynamic [23] and comparison study by using the social comparison theory [24]. Actually, in vent collective behavior, how individual's attitude evolves is restricted by its conformity coefficient and neighbors' attitude. This restriction includes not only interacting imitation or learning but also other nonlinear couplings, such as emotion abreaction. This paper designs a kind of blended learning rules in terms of formula (3). In addition, to estimate whether the system achieves synchronization, the criterion is given as

$$
r=1-\sqrt{\frac{1}{N} \sum_{i=1}^{N}\left(\dot{x}_{i}(t)-\dot{X}(t)\right)^{2}},
$$




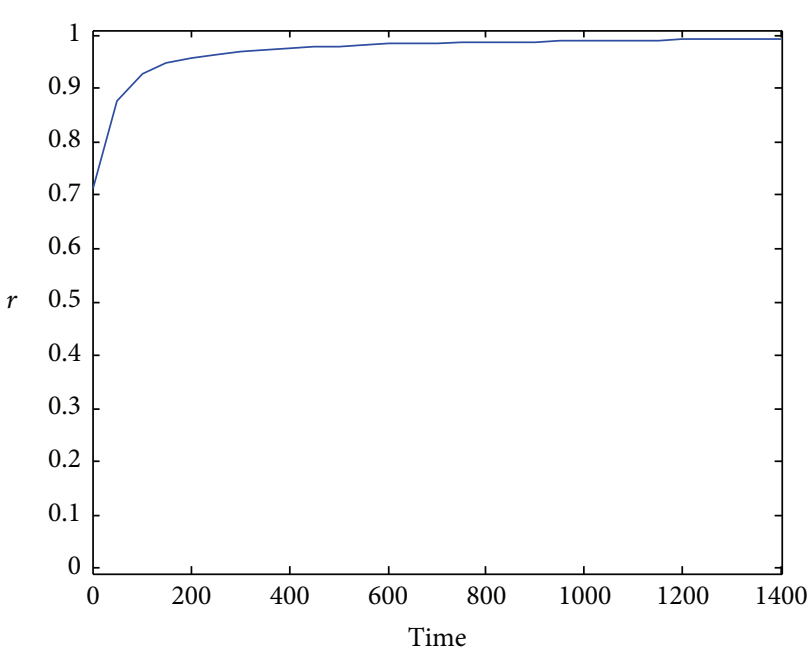

(a)

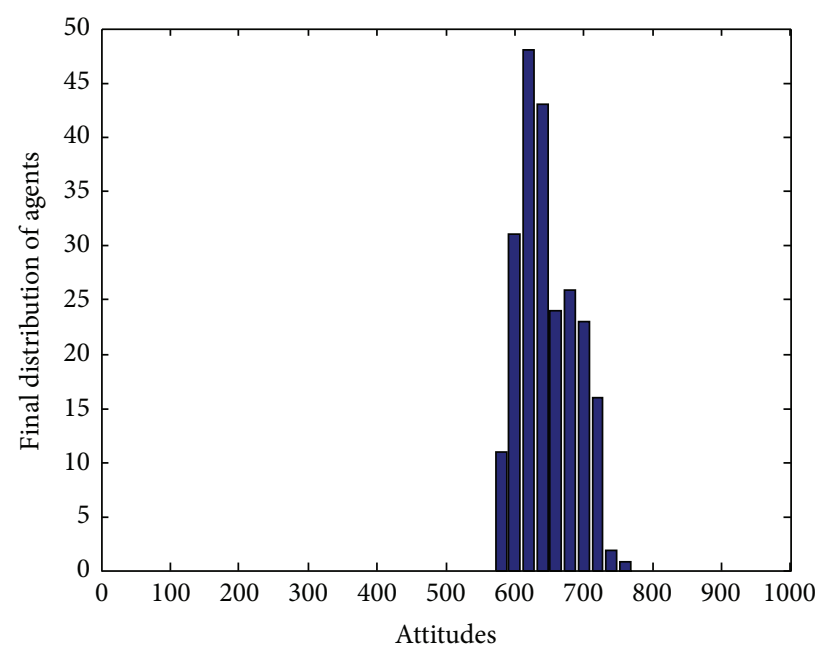

(c)

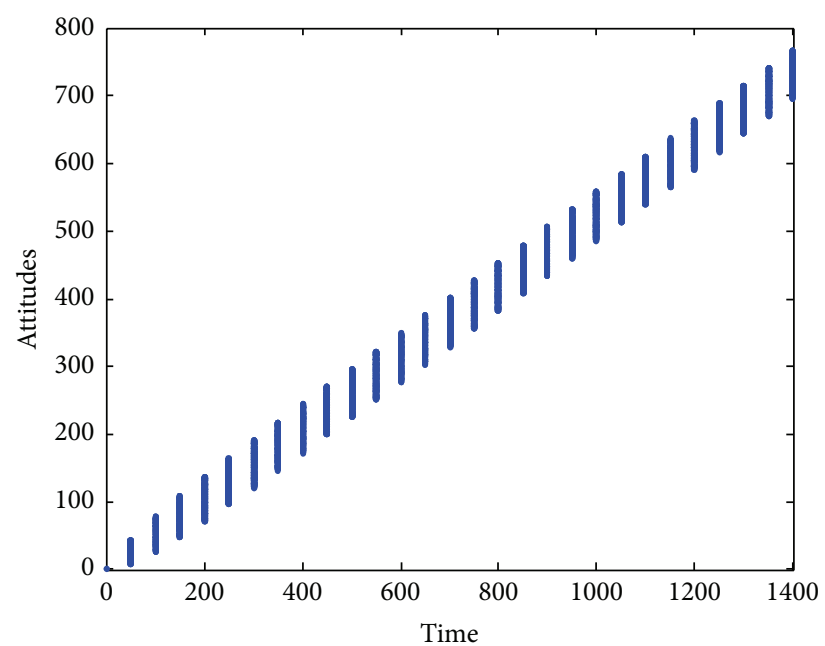

(b)

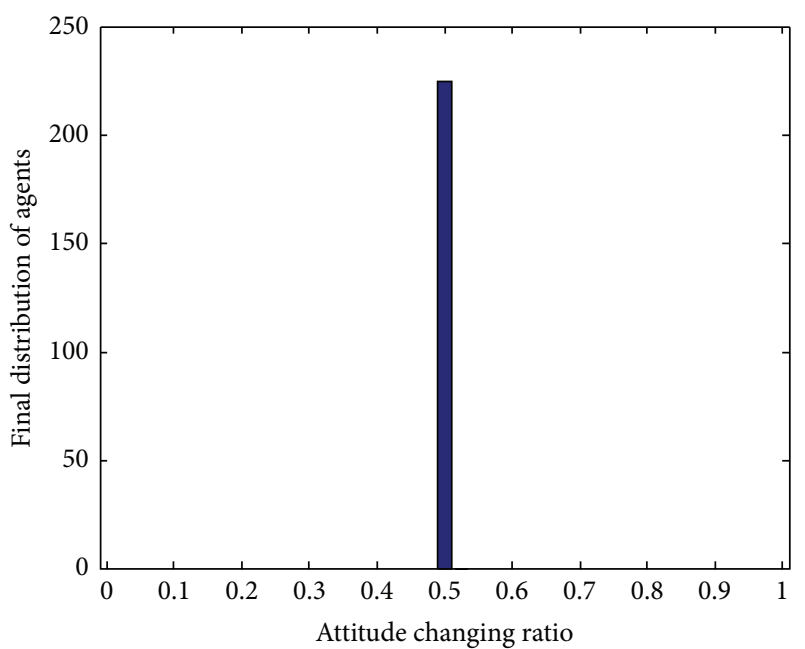

(d)

FiguRE 8: Results of simulation system with $K=1.2$. (a) is the subfigure which shows synchronization criterion $r$ changes with time; (b) is the subfigure which shows group attitudes $x(t)$ versus time; (c) is the histogram of the final distribution of agents versus group attitudes $x(t)$; (d) is the histogram of the final distribution of agents versus attitude changing ratio $\dot{x}_{i}(t)$.

where $\dot{X}(t)$ is the average of $\dot{x}_{i}(t)$. Whether $r$ is close or equal to 1 explains whether the system achieves partial or complete synchronization.

Another notice is that $g(c)$ is a normal distribution function, namely, $c \sim N\left(c_{0}, \sigma^{2}\right)$. When $c$ becomes bigger, the conformity coefficient will be bigger, and individual is more inclined to change its attitude by influence of consensus. When $\sigma^{2}$ becomes bigger, individual's attitude goes to heterogeneity more clearly (Figure 1). In this paper, $h(\alpha)$ and $f(w)$ have the same law with $g(c)$.

\section{Simulation and Results}

In this paper, we use the NetLogo software to make a twodimensional lattice whose size is $15 \times 15$. Let $c_{i} \sim N\left(c_{0}, \sigma^{2}\right)$; by taking $c_{0}=0.5$ and $\sigma=0.17$, we guarantee that the most values of care between 0 and 1 . Furthermore, we only take $c_{i} \in[0,1]$ in this paper, so $c$ is almost always a positive value. When $c=0$, the individual is very stubborn; when $c=1$, the individual will change his opinion according to others completely. Assume that the initial attitude $x_{i}(0)$ obeys uniform distribution $x_{i}(0) \sim$ Uniform $(0,1)$. The coupling effects between individuals' attitude evolve in accordance with (3).

In this paper, we will study three different cases which are global-coupling and homogeneous group, local-coupling and homogeneous group, and local-coupling and heterogeneous group. For global-coupling group, every individual is linked with all of the other individuals. However, the individual is only linked with its neighbors (e.g., nearest neighbors) in local-coupling group. We will simplify the parameters of $w_{i}$ and $\alpha_{j}$ as constant in homogeneous group but set them to obey uniform distribution in heterogeneous group.

To verify the effect of established model and the result of Section 3, we will reveal the process of consensus 


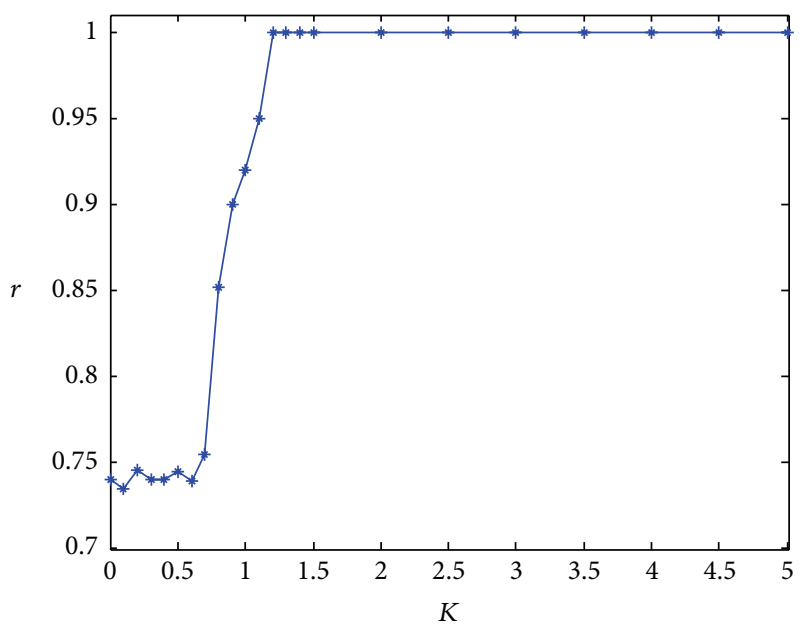

FIgURE 9: When group is local-coupling, the threshold $K_{c}$ is about 1.2 for consensus synchronization in vent collective behavior.

synchronization in global-coupling and homogeneous group of vent collective behavior firstly. And, then, we change the condition of global-coupling to local coupling to find the laws of synchronous consensus. At last, we will find the deep laws for more complex situation of heterogeneous parameters.

5.1. Threshold of Consensus Synchronization in GlobalCoupling and Homogeneous Group. Figures 2(a) and 2(b) are the subfigures which show that synchronization criterion $r$ and collective attitudes $x(t)$ change with time, respectively; Figures 2(c) and 2(d) are the histograms of the distribution of attitude $x(t)$ and the distribution of attitude changing ratio $\dot{x}_{i}(t)$, respectively. From the four subfigures of Figure 2, we can see when $K=0.5$, neither the attitude nor the attitude changing ratio of groups has not reached the synchronization status.

As can be seen from Figure 3, when $K=1$, the attitude changing ratio of vent collective behavior has almost reached the synchronization status; then $r \approx 1$. The attitude changing ratio is stabilized at about 0.5 to 0.6 . But from Figure 3(c) we can see that there are still differences in group opinion; that is, group attitude is not consistent.

Setting $K=2$, we find that not only the attitude but also the attitude changing ratio of groups is close to the synchronization status (Figure 4). With increasing the value of $K$, we find that as $K$ increases, the attitude and the attitude changing ratio tend to be more synchronous (Figure 5).

Figures 2-5 show that, with the increasing of $K$, the system's capability of synchronization also increases. Figure 6 gives the results of $r$ changing with the $K$, where the simulation time is 1500 , and every dot in the figure is the average value of 5 experiments. Corresponding with the conclusion in Section 3, there is a threshold of consensus synchronization in vent collective behavior $K_{c} \approx 0.6$. When $K>K_{c}$, the system reaches synchronization.

5.2. Threshold of Consensus Synchronization in Local-Coupling and Homogeneous Group. In reality, the individual is often not influenced by the whole group to change its attitude but is influenced only by their adjacent small groups. To reflect this fact, we further study the threshold of consensus synchronization in local-coupling group by simulation experiments.

We can see from Figure 7 that when $K=1$, neither the attitude nor the attitude changing ratio of groups has not reached the synchronization status. Figure 8 shows the results of simulation system with $K=1.2$. Although the attitude changing ratio of vent collective behavior has reached the synchronization status, the group attitude is not consistent. Compared with Section 5.1, we find that, with the same parameters, the process of consensus synchronization is delayed to the global-coupling situation.

Figure 9 further reveals the above law. In global-coupling case, the system can basically be synchronized when $K>0.7$, but, in the case of local-coupling, only when $K>1.2$, the system can achieve synchronization. Obviously, this result is not corresponding with [25-27]. This is because there are many factors that may influence the synchronization of consensus, such as the coupling strength $K$ and the number of neighbors. Though Strogatz and Mirollo proved that synchronization was not possible in the thermodynamic limit [26] and similar conclusions were reached by Daido [27], they also point out that the system will be an attainable synchronization for a finite $N$ provided $K$ is sufficiently large. Different from the previous studies [25-27], in this paper, the system is two-dimensional lattice but not one-dimensional chain, the probability distribution of natural frequencies $c$ is also somehow different, and the local-coupling is not the nearest neighbors but is more widely ranging. We think all of these differences may result in the different results.

\subsection{Threshold of Consensus Synchronization in Local-Coupling} and Heterogeneous Group. Finally, we study the threshold of consensus synchronization in the conditions of local coupling, and the individual's influence ability $\alpha$ and acceptance influence ability $w$ are heterogeneous.

Figure 10 shows the results of heterogeneous parameters for individuals. Set that $\alpha$ and $w$ satisfy the normal distribution whose mathematical expectation is 0.5 and variance is 0.17 . Obviously, as can be seen from Figure 10, the system cannot achieve synchronization. The reason is that $\alpha$ and $w$ are too small to affect the system to reach the threshold of consensus synchronization.

Adjusting the mathematical expectation to 1.5 and maintaining variance to be unchanged, we can get the results shown as in Figure 11. The system achieves synchronization now. The reason for this result is that, with the increasing of the mean value of $\alpha$ and $w$, their effects are also increased. When the value of $\alpha$ and $w$ reaches the threshold which can achieve synchronization, the consensus synchronization emerges.

\section{Conclusions}

When the social contradiction and public animosity reach some degree, an emergency usually causes the outbreak of vent collective behavior. The essence of vent collective behavior is emergence of synchronization. Usually, synchronous 


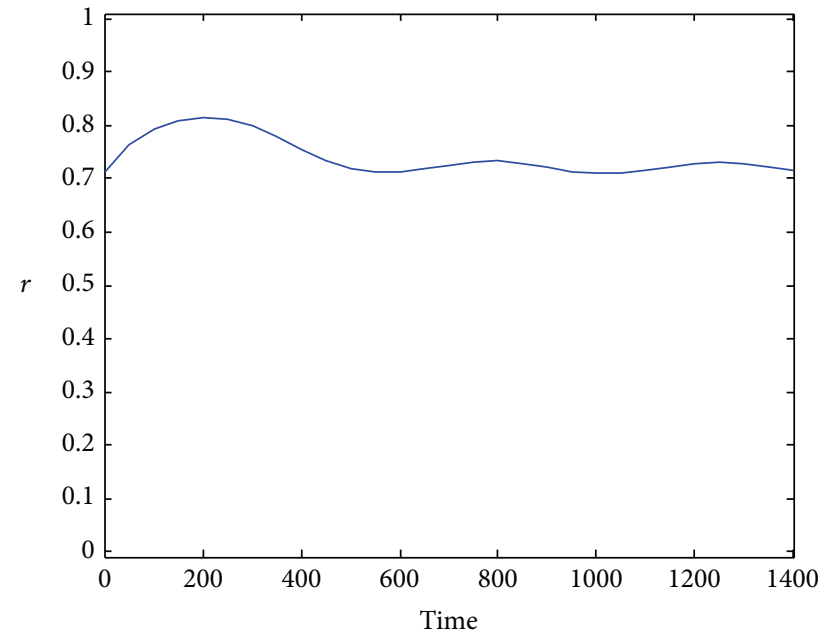

(a)

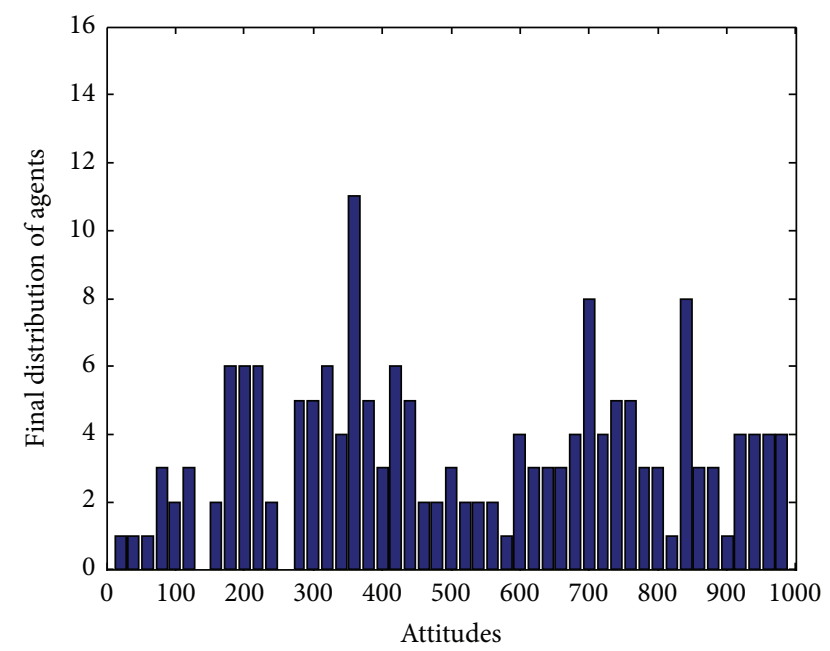

(c)

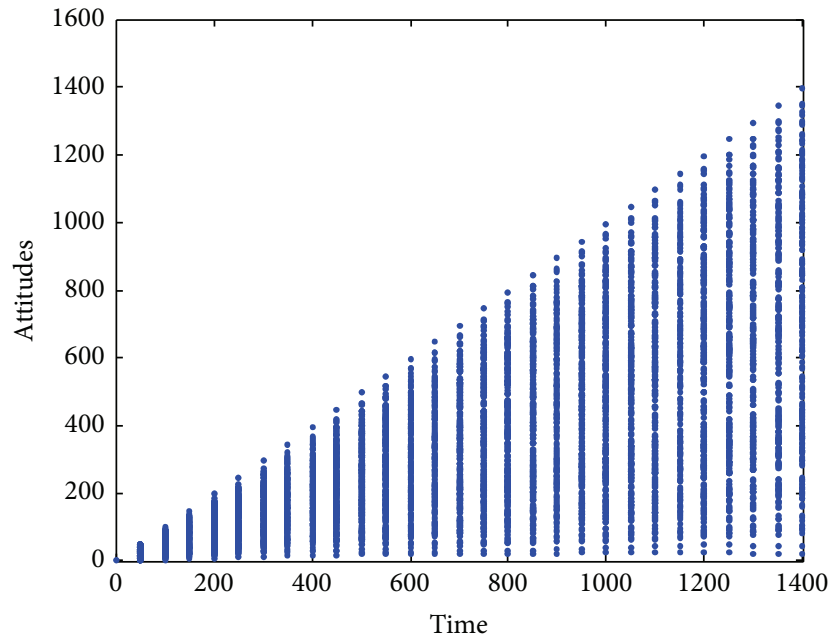

(b)

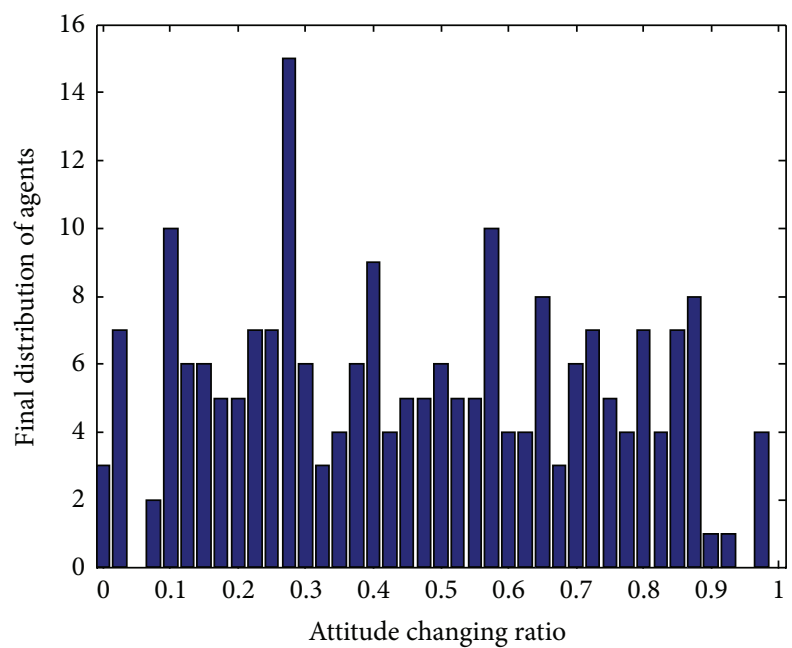

(d)

FIGURE 10: The simulation results with heterogeneous parameters, where $\alpha$ and $w$ satisfy the normal distribution whose mathematical expectation is 0.5 and variance is 0.17 . (a) is the subfigure which shows synchronization criterion $r$ changes with time; (b) is the subfigure which shows group attitudes $x(t)$ versus time; (c) is the histogram of the final distribution of agents versus group attitudes $x(t)$; (d) is the histogram of the final distribution of agents versus attitude changing ratio $\dot{x}_{i}(t)$.

behavior will occur with a certain condition. So, what is the synchronous condition of vent collective behavior? To answer this question, we propose a mathematic model corresponding to the reality. Using the mean field theory and multiagent simulation method, we study three situations which are global-coupling and homogeneous group, local-coupling and heterogeneous group, and local-coupling and homogeneous group. Along with the increasing of complexity of model, we carry out series simulation experiments. The results show that there is a threshold $K_{c}$ for consensus synchronization in global-coupling and homogeneous group and the threshold $K_{c}$ is related to the original distribution of conformity coefficient. When $K>K_{c}$, consensus of vent collective behavior will achieve partial or complete synchronization.
When $K \leq K_{c}$, consensus cannot achieve synchronization. For local-coupling and homogeneous group, the process of consensus synchronization is delayed compared with the global-coupling situation for the same parameters. For localcoupling and heterogeneous group, with the increasing of the mean value of $\alpha$ and $w$, their effects are also increased; when the value of $\alpha$ and $w$ reaches the threshold which can achieve synchronization, the consensus synchronization emerges.

\section{Conflict of Interests}

The authors declare that there is no conflict of interests regarding the publication of this paper. 


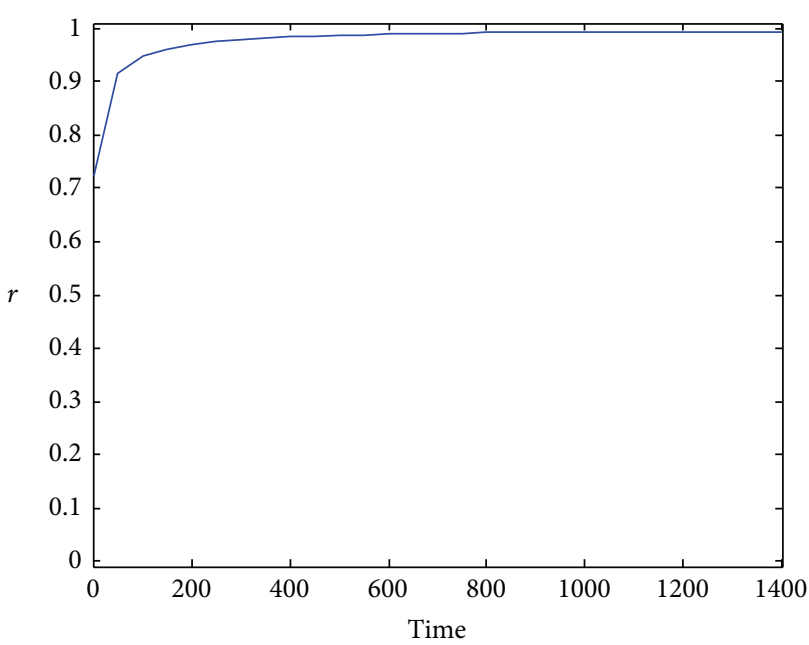

(a)

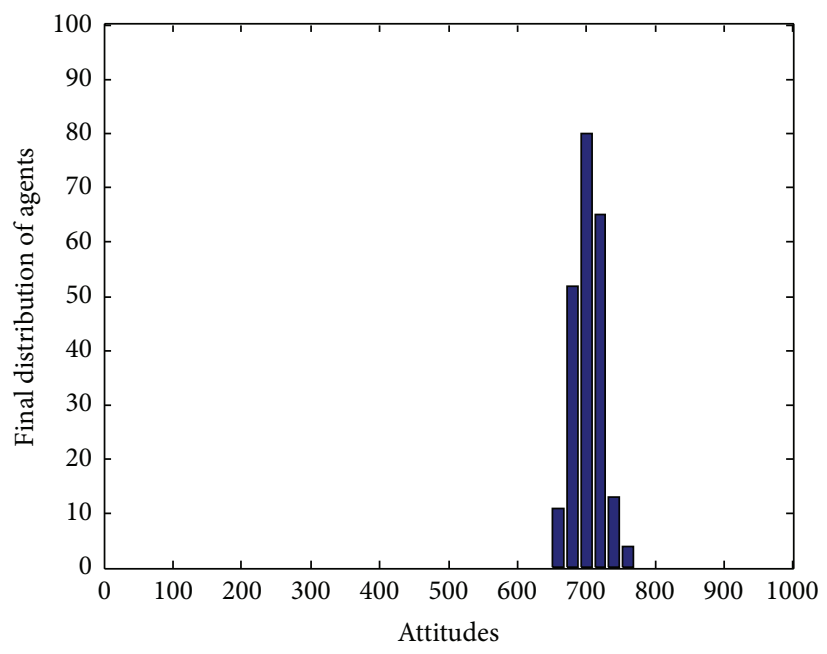

(c)

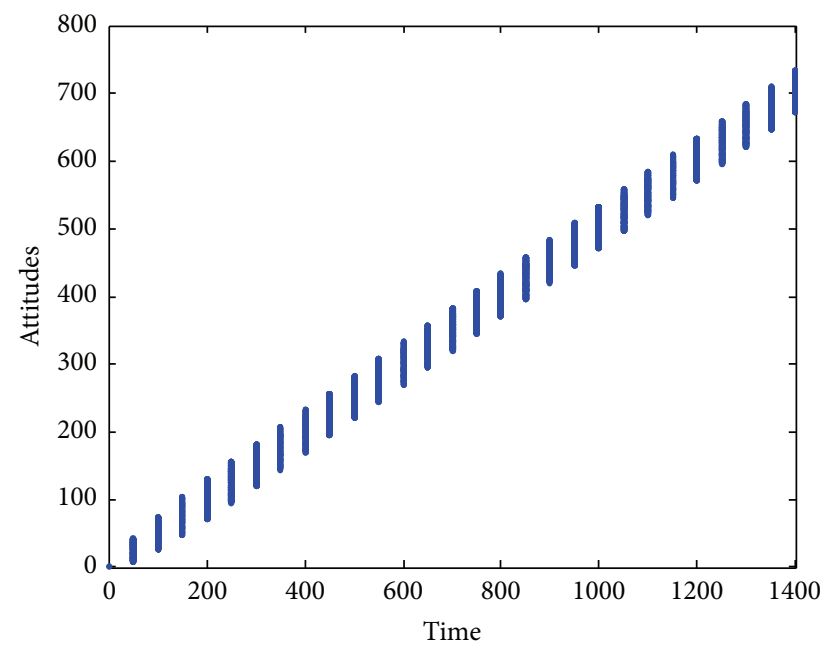

(b)

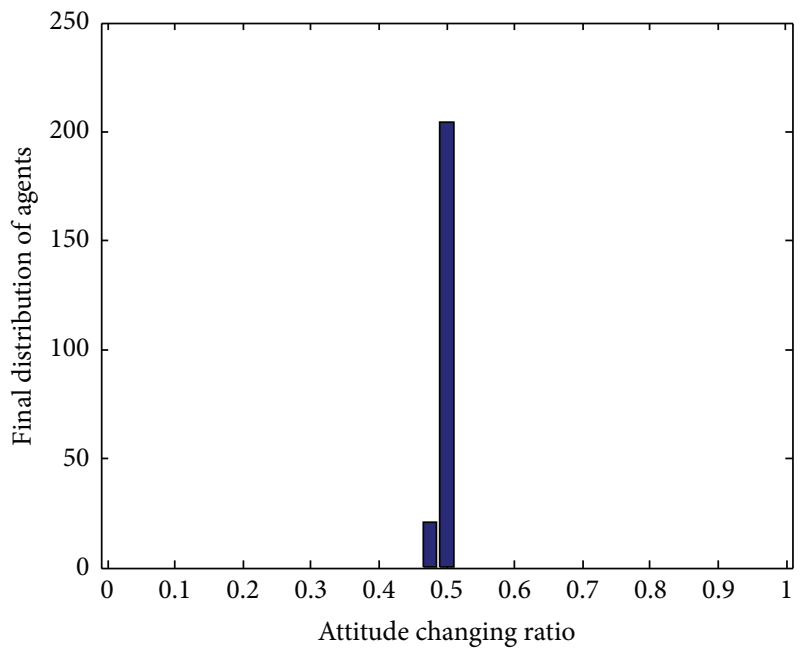

(d)

FiguRE 11: The simulation results with heterogeneous parameters, where $\alpha$ and $w$ satisfy the normal distribution whose mathematical expectation is 1.5 and variance is 0.17 . (a) is the subfigure which shows synchronization criterion $r$ changes with time; (b) is the subfigure which shows group attitudes $x(t)$ versus time; (c) is the histogram of the final distribution of agents versus group attitudes $x(t)$; (d) is the histogram of the final distribution of agents versus attitude changing ratio $\dot{x}_{i}(t)$.

\section{Acknowledgments}

The authors gratefully acknowledge helpful suggestions from the anonymous reviewers. This research was supported by the Specialized Research Fund for the Doctoral Program of Higher Education of China (Grant no. 20130142110051), the National Natural Science Foundation of China (Grant no. 71101047) and Youth Science Foundation of Hubei University of Economics, China (Grant no. XJ201301).

\section{References}

[1] A. Arenas, A. Díaz-Guilera, J. Kurths, Y. Moreno, and C. Zhou, "Synchronization in complex networks," Physics Reports, vol. 469, no. 3, pp. 93-153, 2008.

[2] N. E. Friedkin and E. C. Johnsen, "Social influence networks and opinion change," Advances in Group Processes, vol. 16, no. 1, pp. 1-29, 2003.
[3] B. John and E. Buck, "Toward a functional interpretation of synchronous flashing by fireflies," The American Naturalist, vol. 112, no. 985, pp. 471-492, 1978.

[4] E. Sismondo, "Synchronous, alternating, and phase-locked stridulation by a tropical katydid," Science, vol. 249, no. 4964, pp. 55-58, 1990.

[5] A. Pikovsky, M. Rosenblum, and J. Kurths, Synchronization: A Universal Concept in Nonlinear Sciences, Cambridge University Press, 2001.

[6] D. Y. Li, K. Liu, Y. Sun, and M. C. Han, "Emergent computation: virtual reality from disordered clapping to ordered clapping," Science in China F: Information Sciences, vol. 51, no. 5, pp. 449459, 2008.

[7] Q. X. Zeng and W. Li, Group Events: Information Dissemination and Government Countermeasures, China Book Press, Beijing, China, 2010, (Chinese).

[8] D. T. Campbell, "On the conflicts between biological and social evolution and between psychology and moral tradition," American Psychologist, vol. 30, no. 12, pp. 1103-1126, 1975. 
[9] S. C. Wright, D. M. Taylor, and F. M. Moghaddam, "Responding to membership in a disadvantaged Group: from acceptance to collective protest," Journal of Personality and Social Psychology, vol. 58, no. 6, pp. 994-1003, 1990.

[10] J. Drury, C. Coucking, S. Reicher et al., "Cooperation versus competition in a mass emergency evacuation: a new laboratory simulation and a new theoretical model," Psychonomic Society, vol. 41, no. 3, pp. 957-970, 2009.

[11] O. Alvarez-Llamoza, K. Tucci, M. G. Cosenza, and M. Pineda, "Random global coupling induces synchronization and nontrivial collective behavior in networks of chaotic maps," European Physical Journal: Special Topics, vol. 143, no. 1, pp. 245-247, 2007.

[12] H. W. Yu and Y. F. Zheng, "Dynamic behavior of multi-agent systems with distributed sampled control," Acta Automatica Sinica, vol. 38, no. 3, pp. 357-365, 2012 (Chinese).

[13] W. S. Yan, J. B. Li, and Y. T. Wang, "Consensus for damaged multi-agent systems," Acta Automatica Sinica, vol. 38, no. 11, pp. 1880-1884, 2012 (Chinese).

[14] J. G. Jiang, G. F. Zhang, N. Xia, and Z. P. Su, “Task oriented coalition formation strategy based on rational agents," Acta Automatica Sinica, vol. 34, no. 4, pp. 478-481, 2008 (Chinese).

[15] T. Takahashi and H. Shiizuka, "Refuge behavior simulation by network model," Memoirs of Kougakuin University, vol. 73, pp. 213-220, 1992.

[16] M. Le Bars, J. M. Attonaty, S. Pinson, and N. Ferrand, "An agent-based simulation testing the impact of water allocation on farmers' collective behaviors," Simulation, vol. 81, no. 3, pp. 223-235, 2005.

[17] C.-Y. Huang, P.-J. Tzou, and C.-T. Sun, "Collective opinion and attitude dynamics dependency on informational and normative social influences," Simulation, vol. 87, no. 10, pp. 875-892, 2011.

[18] L. P. Kadanoff, "More is the same; phase transitions and mean field theories," Journal of Statistical Physics, vol. 137, no. 5-6, pp. 777-797, 2009.

[19] "Self-entrainment of a population of coupled non-linear oscillators," in International Symposium on Mathematical Problems in Theoretical Physics, Y. Kuramoto and H. Arakai, Eds., vol. 39 of Lecture Notes in Physics, pp. 420-422, Springer, New York, NY, USA, 1975.

[20] J. A. Acebrón, L. L. Bonilla, C. J. P. Vicente, F. Ritort, and R. Spigler, "The Kuramoto model: a simple paradigm for synchronization phenomena," Reviews of Modern Physics, vol. 77, no. 1, pp. 137-185, 2005.

[21] Y. Kuramoto, Chemical Oscillations, Waves, and Turbulence, Springer, Berlin, Germany, 1984.

[22] S. H. Strogatz, "From Kuramoto to Crawford: exploring the onset of synchronization in populations of coupled oscillators," Physica D: Nonlinear Phenomena, vol. 143, no. 1-4, pp. 1-20, 2000.

[23] W. Jorgen, Evolutionary Game Theory, MIT Press, Cambridge, Mass, USA, 1995.

[24] N. Fridman and G. A. Kaminka, "Modeling pedestrian crowd behavior based on a cognitive model of social comparison theory," Computational and Mathematical Organization Theory, vol. 16, no. 4, pp. 348-372, 2010.

[25] J. L. Rogers and L. T. Wille, "Phase transitions in nonlinear oscillator chains," Physical Review E, vol. 54, no. 3, Article ID R2193, 1996.

[26] S. H. Strogatz and R. E. Mirollo, "Phase-locking and critical phenomena in lattices of coupled nonlinear oscillators with random intrinsic frequencies," Physica D: Nonlinear Phenomena, vol. 31, no. 2, pp. 143-168, 1988.

[27] H. Daido, "Lower critical dimension for populations of oscillators with randomly distributed frequencies: a renormalizationgroup analysis," Physical Review Letters, vol. 61, no. 2, pp. 231234, 1988. 


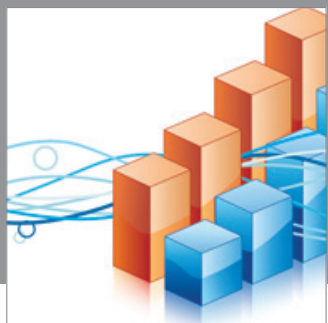

Advances in

Operations Research

mansans

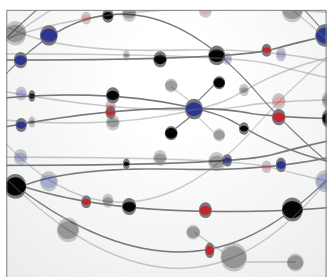

The Scientific World Journal
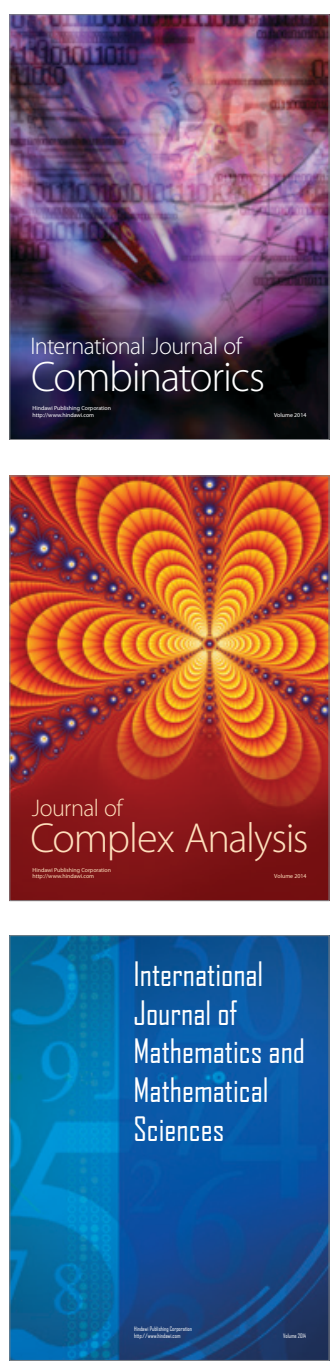
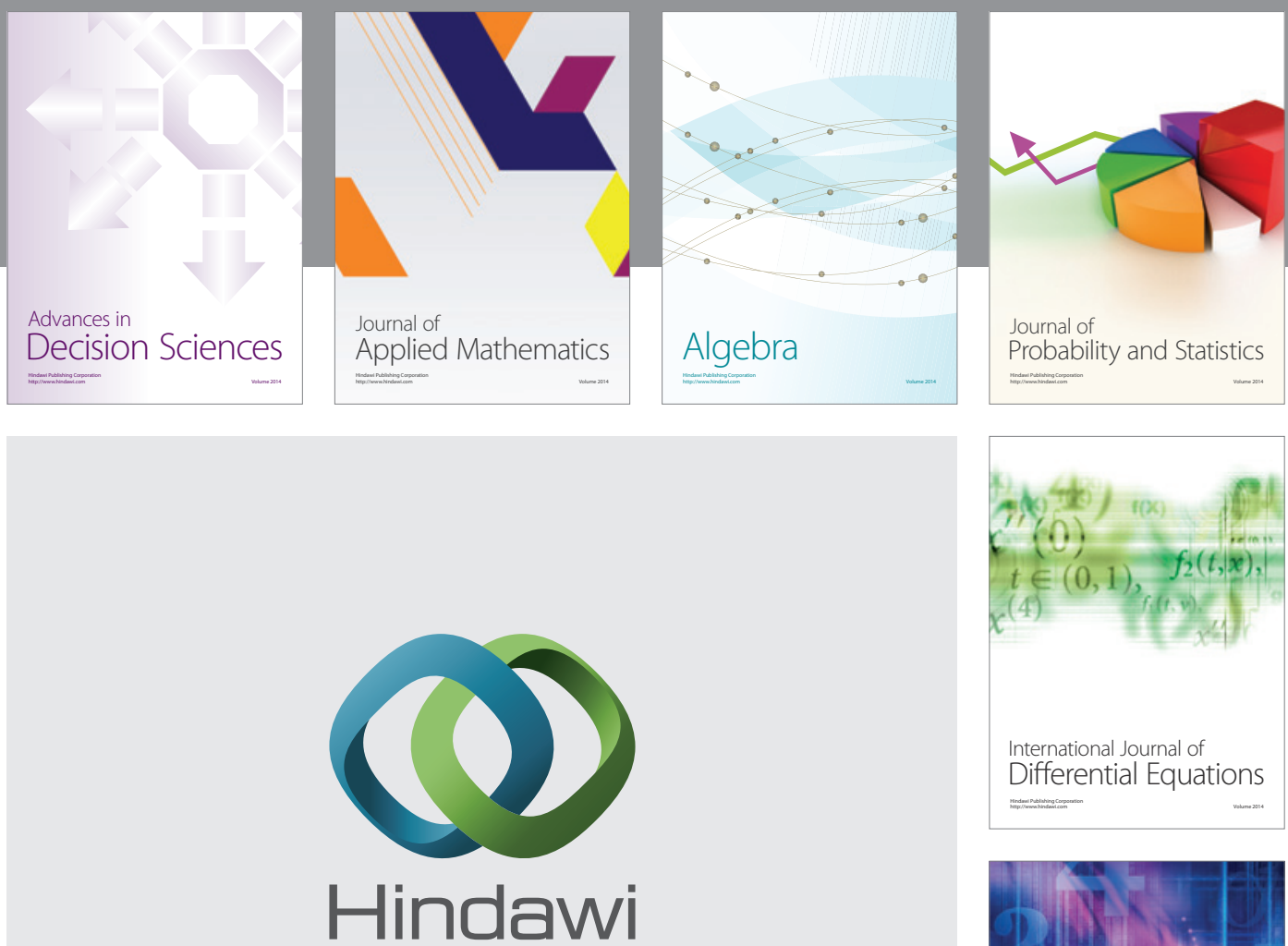

Submit your manuscripts at http://www.hindawi.com
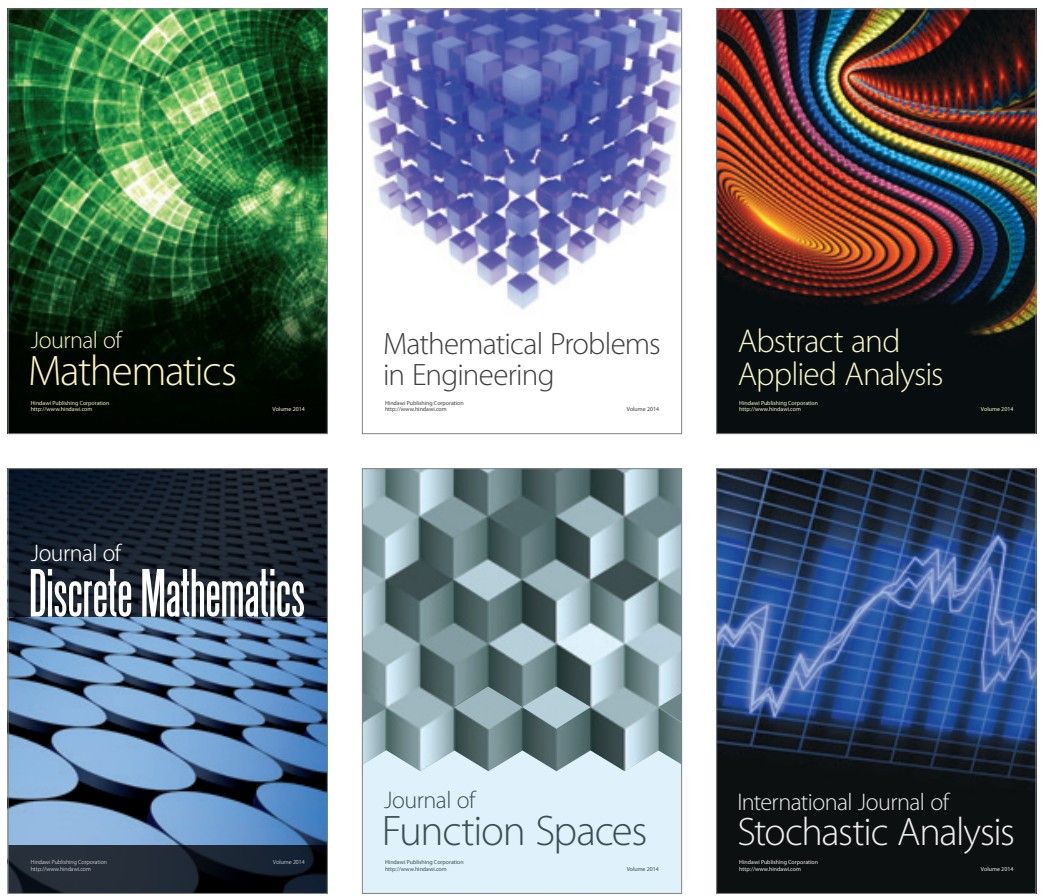

Journal of

Function Spaces

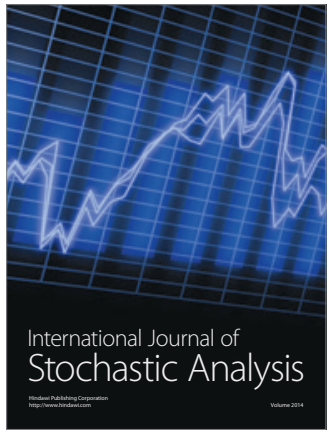

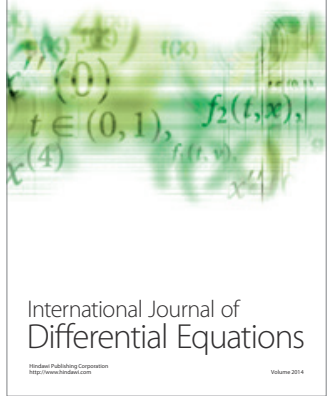
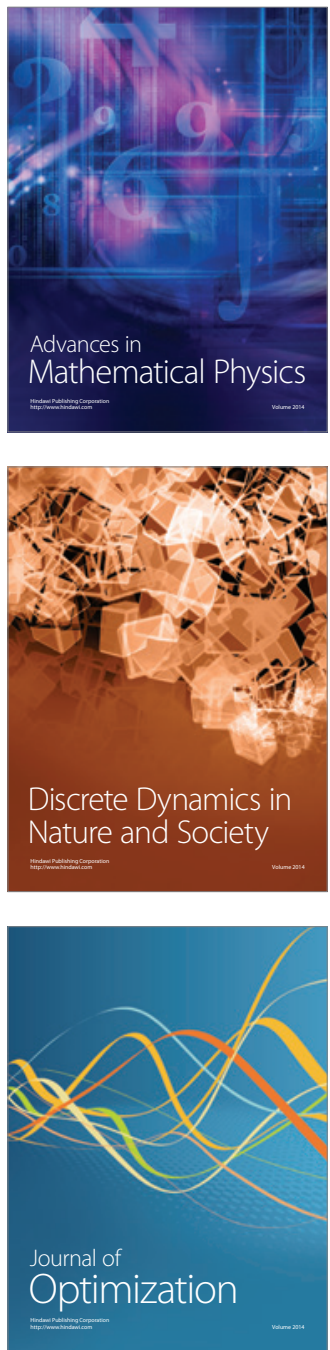